\title{
Visualización y detección cuantitativa del cáncer de piel utilizando sensores de temperatura por resistencia
}

\section{Quantitative visualization and detection of skin cancer using resistance temperature sensors}

\author{
Raul E. Franceschi F. ${ }^{*}$, Marlen Elisa ${ }^{1}$, Veleiro Herrera ${ }^{2}$, Haydée Osorio ${ }^{3}$ \\ ${ }^{1}$ Licenciatura en Ingeniería Mecánica, Facultad de Ingeniería Mecánica, Universidad Tecnológica de Panamá \\ ${ }^{2}$ Licenciatura en Ingeniería Naval, Facultad de Ingeniería Mecánica, Universidad Tecnológica de Panamá \\ ${ }^{3}$ Facultad de Ingeniería Civil, Universidad Tecnológica de Panamá
}

\begin{abstract}
Resumen FUNDACÁNCER indica que el cáncer de piel ocupa el cuarto lugar entre los de mayor incidencia en Panamá. Al investigar los métodos de diagnóstico actuales, se ha descubierto que todavía es una prueba puramente cualitativa, basada únicamente en la inspección visual. Después de un efecto de enfriamiento sobre la epidermis, las lesiones benignas tienen una recuperación térmica similar a la piel normal, mientras que la recuperación térmica de la lesión maligna se termoregula en un intervalo de tiempo menor. El objetivo principal es diseñar un dispositivo costo efectivo para añadir un valor cuantitativo a los métodos actuales mediante el contacto físico sobre la piel.
\end{abstract}

Palabras clave Cáncer de piel, melanoma, efecto de enfriamiento, termoregulación, matriz de termistores, mapa térmico, valor cuantitativo.

\begin{abstract}
FUNDACÁNCER indicates that skin cancer ranks fourth among the highest incidence in Panama. By investigating current diagnostic methods, it has been discovered that it is still a purely qualitative test, based solely on visual inspection. After a cooling effect on the epidermis, the benign lesions have a thermal recovery similar to normal skin, while the thermal recovery of the malignant lesion is thermoregulated in a shorter time interval. The main objective is to design a cost-effective device to add a quantitative value to current methods through physical contact on the skin.
\end{abstract}

Keywords skin cancer, melanoma, cooling effect, thermoregulation, thermistors matrix, heat map, quantitive value.

* Corresponding author: raul.franceschi@ outlook.com

\section{Introducción}

En los últimos años se ha dado un aumento en la tasa del cáncer de piel. En América Latina se presenta en los países que se encuentran cerca de la línea ecuatorial, de los cuales Panamá forma parte. La causa aparenta ser obvia, mayor exposición en tiempo y en intensidad a los rayos ultravioletas durante la exposición al sol en las playas, piscinas y días de campo. Sin embargo, no se toma en cuenta la exposición que se tiene durante las actividades cotidianas [1].

En la culminación de la XV Campaña de Prevención y Detección Temprana del Cáncer de Piel, organizada por la Fundación Pro Enfermos del Cáncer (FUNDACÁNCER) con el apoyo de la Asociación Panameña de Dermatología y de la Dra. Rosa España de Villanueva, jefa de la clínica de piel Irene Escoffery del Instituto Oncólogico Nacional, de un total de 259 casos atendidos, 48 casos fueron detectados con cáncer de piel [2].

Luego del desarrollo de la jornada "Soy Skin Checker" del laboratorio La Roche-Posay, con el apoyo de otros laboratorios tales como: Sesderma, Siedfried, Cantabria, Roemmers, Bioderma, Avene, Quimifar y Suiphar; la directora ejecutiva de FUNDACÁNCER, Alexandra Castro Novey, en el acto de cierre de la XVI campaña, expresó que de 1,069 personas atendidas, lograron detectar 109 casos de cáncer de piel [3].

En el 2018, con el apoyo de la Dra. M. Quelquejeu, Dr. R. Villanueva, Dra. D. Tejada, Dr. J. González, Dr. F. Díaz, Dra. G. Archbold y Dra. S. Sánchez, médicos que formaron parte de la XVII campaña, se dió una detección de 17 nuevos casos de cáncer de piel de un total de 328 personas atendidas [4].

A partir de estos resultados, FUNDACÁNCER indica que el cáncer de piel ocupa el cuarto lugar entre los de mayor incidencia en el país, el cual es superado por el de próstata, mamá y cérvico uterino. Por lo tanto, el cáncer de piel es uno de los tumores más frecuentes en Panamá.

\section{Planteamiento del problema}

Panamá es un país en el cual, prácticamente, hay sol los 365 días del año; en consecuencia, la importancia de protegerse de 
los efectos nocivos del mismo es todavía mayor. El sol y sus efectos son un factor de riesgo en el desarrollo anormal de lunares y manchas en la piel, que luego pueden tornarse en afecciones graves como el melanoma o el cáncer de piel.

El melanoma es inofensivo si se detecta temprano; sin embargo, todavía reclama cientos de miles de vidas alrededor del mundo anualmente. La investigación de clínicas en los EE.UU. muestra que solo alrededor de 1 de 50 biopsias de la piel en realidad tenían cáncer. Estos dos factores significan que el melanoma se diagnostica erróneamente $\mathrm{y}$, a menudo, se detecta demasiado tarde. Al investigar los métodos de diagnóstico actuales se ha descubierto que todavía es una prueba puramente cualitativa, basada únicamente en la inspección visual.

Esta es la razón por la cual se está innovando con nuevas tecnologías para la rápida detección de melanomas, especialmente herramientas no invasivas de diagnóstico tales como la dermatoscopia [5]. La dermatoscopia permite identificar estructuras de la piel no identificables a simple vista. Datos precisos sobre la temperatura del cuerpo humano y la piel pueden proporcionar una gran cantidad de información sobre los procesos responsables de la termorregulación, en particular sobre la desviación de las condiciones normales, a menudo causada por la enfermedad.

C. Herman y M. P. Cetingul en el 2010 desarrollaron una técnica de visualización y detección cuantitativa del cáncer de piel utilizando imágenes térmicas dinámicas, en otras palabras, llegaron a la conclusión de que la actividad metabólica de la lesión puede ser detectada por imágenes infrarrojas. Su método demostró que los melanomas se pueden identificar en una etapa temprana al medir una respuesta transitoria térmica de la piel después de aplicar una excitación de enfriamiento.

Con base en las imágenes térmicas dinámicas, observaron una diferencia de temperatura durante la recuperación térmica en la región de la lesión maligna, mientras que las lesiones pigmentadas benignas exhibieron una recuperación térmica similar a la piel sana circundante. Esto sugiere que las lesiones benignas tienen una recuperación térmica similar a la piel normal, mientras que la recuperación térmica de la lesión maligna es diferente [6].

Esta técnica de imágenes térmicas dinámicas ofrece ventajas e inconvenientes, representa un compromiso entre la eficacia y la precisión frente a la facilidad de uso y el costo. Por lo tanto, el objetivo principal de esta investigación es diseñar un dispositivo costo efectivo para añadir un valor cuantitativo a los métodos actuales mediante el contacto físico sobre la piel; con el fin, de optimizar su precisión y disminuir el descubrimiento tardío de los melanomas.

\section{Diseño del prototipo}

El próposito principal del dispositivo es ayudar a los médicos para detectar melanomas mediante la creación de un mapa térmico de la región de interés en la piel. Proporcionar una medida cuantitativa para mejorar el método de diagnóstico, el cual es puramente cualitativo e ineficiente.

El diseño consiste en un transductor, un convertidor analógico-digital y un procesador de software. El transductor es una matriz $2 \mathrm{X} 2$ de termistores que son colocados en la piel con el propósito de tomar medidas de la temperatura corporal en tiempo real.

Como transductor se utilizó el sensor de temperatura por resistencias denominado DHT22, el cual es un sensor digital de temperatura que utiliza un termistor para medir la superficie de la piel. Este transmite una señal digital a $0.5 \mathrm{~Hz}$ cada 2 segundos, y realiza lecturas de temperatura desde $\operatorname{los}-40^{\circ} \mathrm{C}$ hasta los $80^{\circ} \mathrm{C}$ con $\pm 5^{\circ} \mathrm{C}$ de precisión [7].

A medida que la temperatura de cada termistor varía, el voltaje a través del circuito cambia, enviando una señal a través de un circuito de acondicionamiento para filtrar y ampliar la señal antes de ser digitalizada por un microcontrolador.

El microcontrolador consiste en un Arduino Uno, el cual es una placa basada en ATmega328P. Tiene 14 pines digitales de entrada/salida (de los cuales seis se pueden usar como salidas PWM), seis entradas analógicas, un cristal de cuarzo de 16 $\mathrm{MHz}$, una conexión USB, un conector de alimentación y un botón de reinicio [8].

La señal capatada por el Arduino Uno se envía a MATLAB a través de comunicación serial para ajustarse a las curvas paramétricas del termistor [9].

Inicialmente los sensores realizan un reconocimiento para determinar la temperatura del ambiente. Finalizado el reconocimiento, inicia la primera etapa que consiste en tomar diez muestras con cada sensor durante 40 segundos para determinar la temperatura promedio de la piel.

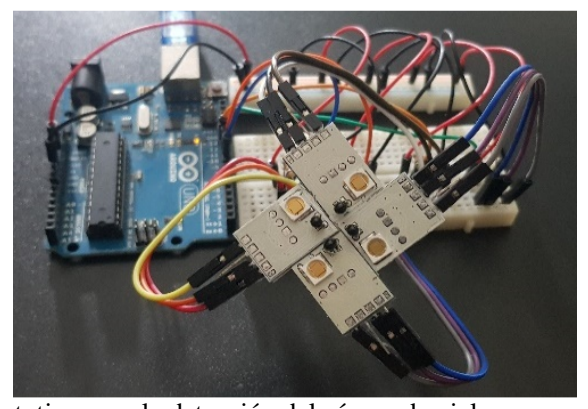

Figura 1. Prototipo para la detección del cáncer de piel.

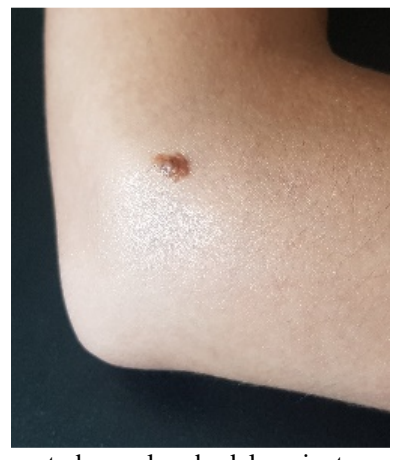

Figura 2. Lesión pigmentada en el codo del paciente. 


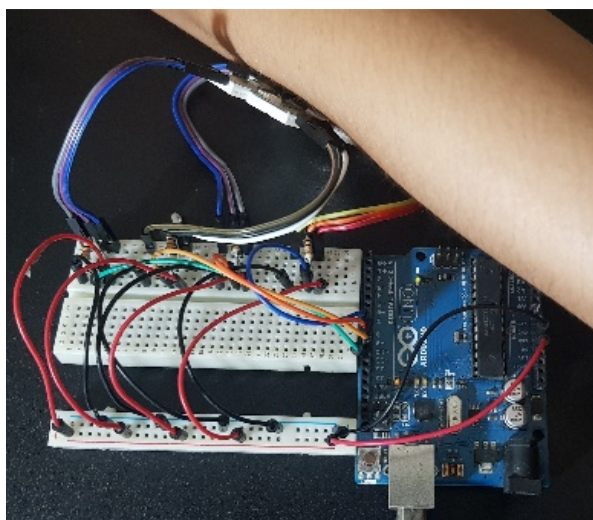

Figura 3. Validación experimental del prototipo.

En la tabla 1 se muestran los valores censados por los cuatros termistores. Analizando los resultados, se determina que la variación de las medidas no es considerable; por lo tanto, podemos inferir que el margen de error en la precisión es insignificante.

TABLA 1. Muestras captadas por la matriz de termistores durante la

\begin{tabular}{|c|c|c|c|c|c|c|c|c|c|c|}
\hline$n$ & 1 & 2 & 3 & 4 & 5 & 6 & 7 & 8 & 9 & 10 \\
\hline$T_{1}$ & 33.6 & 33.8 & 34.3 & 34.3 & 33.7 & 34.0 & 34.4 & 34.5 & 33.7 & 34.0 \\
\hline$T_{2}$ & 34.5 & 34.5 & 33.8 & 34 & 34.5 & 34.6 & 33.8 & 34.1 & 34.6 & 34.6 \\
\hline$T_{3}$ & 33.9 & 34.1 & 34.6 & 34.7 & 33.9 & 34.2 & 34.6 & 34.7 & 33.9 & 34.2 \\
\hline$T_{4}$ & 34.6 & 34.8 & 34.0 & 34.2 & 34.7 & 34.8 & 34.0 & 34.2 & 34.7 & 34.8 \\
\hline
\end{tabular}

Posteriormente, en tiempo real, el software muestra la obtención de los valores de la temperatura y un mapa térmico que refleja los valores promedio obtenidos por los sensores. El objetivo del mapa de calor es que se puedan interpretar con sencillez las zonas de mayor temperatura.

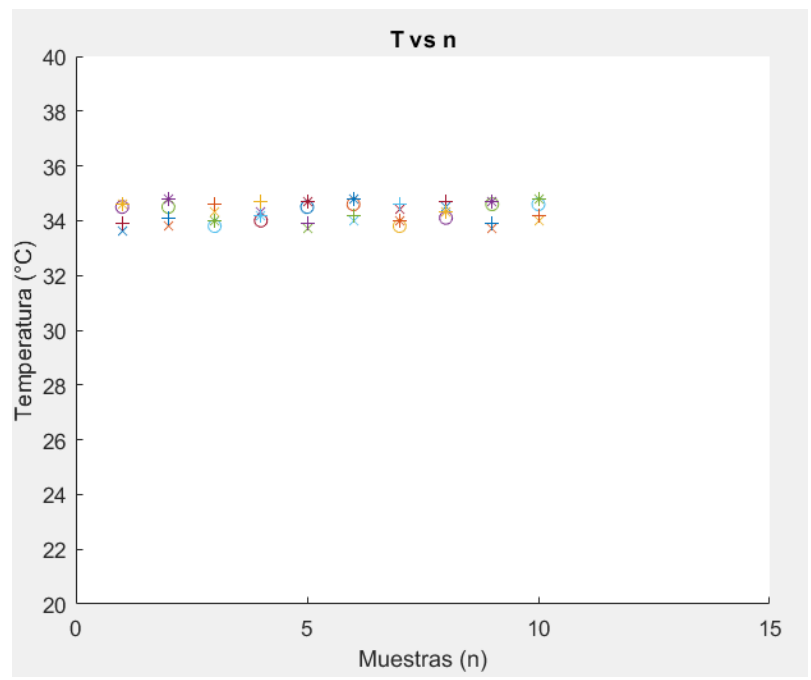

Gráfico 1. Muestras captadas por la matriz de termistores en tiempo real.

$\mathrm{Al}$ analizar los datos obtenidos, el prototipo logra su objetivo; captar datos de la temperatura de la piel con precisión. Por consiguiente, se asimila a los dispositivos que analizan lesiones en la piel.

\section{Funcionamiento}

La fase inicial consiste en monitorear con la matriz de temistores la temperatura del recinto donde se realizarán los exámenes y la superficie de la piel donde está ubicada la lesión pigmentada del paciente.

El protocolo para capturar datos al utilizar los termistores inicia al realizar una medición con los sensores de temperatura por resistencia DHT22. Los datos son almacenados y procesados para generar un mapa térmico de la región de interés.

En la segunda fase, el área de la piel que contiene la lesión se enfría durante 60 segundos. Después del proceso de enfriamiento, se retira la herramienta para permitir que la piel se termoregule durante dos a tres minutos al ser expuesta a la temperatura ambiente. Durante la fase de recuperación, se realizan mediciones nuevamente y los datos son almacenados y procesados en MATLAB.

En la última etapa, el mapa térmico de la dos fases se evalúa con el fin de obtener la distribución de la temperatura de la región de intérés, y se analizan los valores de las temperaturas sensadas por los termistores para determinar cuáles mostraron una diferencia de temperatura significativa durante los minutos en los cuales se aplica la prueba, para una posterior toma de decisiones por parte de los dermatólogos.

\section{Modelo matemático}

El desarrollo del modelo matemático es de suma importancia en el estudio de problemas médicos. En esta sección se describirá el modelo computacional en el cual se ha de simular un melanoma maligno. El volumen de control fue diseñado según las respectivas especificaciones propuestas utilizando el software de diseño asistido por computadora Inventor Professional [10].

La lesión seleccionada para este estudio consiste en un melanoma maligno invasivo. La lesión es superficial tipo de ensanchamiento Breslow con un nivel Clark II. La profundidad del tumor está directamente relacionada con el pronóstico del melanoma. Los tumores más gruesos tienen un mayor acceso a los vasos linfáticos; por lo tanto, pueden llegar a hacer metástasis.

El Breslow describe la profundidad de la penetración de un melanoma en la piel. El nivel de Clark es un sistema de clasificación que describe el nivel de invasión anatómica del melanoma, el nivel II significa que el melanoma ha invadido la segunda capa de la piel, la dermis [11].

El tejido de la piel se modela en cinco capas, vistas desde la superficie son: epidermis, dermis papilar, dermis reticular, capa de grasa y músculo. Estas capas son caracterizadas por un conjunto de propiedades termofísicas, las cuales son necesarias para modelar los procesos de transferencia de calor en la piel humana. Estas propiedades, así como los espesores de las capas 
de la piel se resumen en la tabla 1. La ecuación diferencial de biocalor descrita por Pennes [12] se usa para describir la distribución de temperatura en cada una de las cinco capas de tejido para $(n=1,2,3,4,5)$ :

\begin{tabular}{|c|c|}
\hline$q_{n} C_{n} \frac{\partial T_{n}}{\partial t}=k_{n} \nabla^{2} T_{n}+\rho_{b} C_{b} \omega_{b}\left(T_{b}-T_{n}\right)+Q_{n}$ & (1) \\
\hline$h_{n-1}<y<h_{n}, h_{0}=0$ & \\
\hline
\end{tabular}

Donde $\rho, C, T, k$ y $Q$ denotan la densidad del tejido, el calor específico del tejido, la temperatura del tejido, la conductividad térmica del tejido y la generación de calor metabólico por unidad de volumen, respectivamente. $\rho_{b}, C_{b}, T_{b} y \omega_{b}$ representan la densidad de la sangre, el calor específico de la sangre, la temperatura de la sangre arterial y la tasa de la perfusión de la sangre, respectivamente.

TABLA 2. Espesor de las capas y propiedades termofísicas

\begin{tabular}{|l|c|c|c|c|c|c|}
\hline & $h$ & $C$ & $k$ & $\rho$ & $\omega_{b}$ & $Q$ \\
\hline Epidermis & 0.1 & 3589 & 0.235 & 1200 & 0 & 0 \\
\hline $\begin{array}{l}\text { Dermis } \\
\text { papilar }\end{array}$ & 0.7 & 3300 & 0.445 & 1200 & 0.0002 & 368.1 \\
\hline $\begin{array}{l}\text { Dermis } \\
\text { reticular }\end{array}$ & 0.8 & 3300 & 0.445 & 1200 & 0.0013 & 368.1 \\
\hline Grasa & 2 & 2674 & 0.185 & 1000 & 0.0001 & 368.3 \\
\hline Músculo & 8 & 3800 & 0.51 & 1085 & 0.0027 & 684.2 \\
\hline Lesión & - & 3852 & 0.558 & 1030 & 0.0063 & 3680 \\
\hline
\end{tabular}

La ecuación (1) se resuelve mediante la imposición apropiada de condiciones de contorno en la superficie de la piel, y para la temperatura y flujo de calor en cada capa de la piel. Además se consideró un enfriamiento de la superficie de la piel y una posterior recuperación térmica de la lesión y el tejido sano circundante.

Para lograr esto se asume que para $t<0$ están presentes las condiciones de estado estacionario del tejido y la superficie superior de la piel está expuesta a la condición de contorno a convección natural. Esta solución de estado estacionario sirve como la condición inicial para estudiar los efectos del enfriamiento y recuperación térmica durante la fase de enfriamiento.

En la simulación, la piel se enfría durante un minuto, después de un minuto, la condición de contorno constante de temperatura se retira y la superficie de la piel es nuevamente expuesta a convección natural con el fin de que se permita que la piel regrese a su temperatura original. Este procedimiento se denomina termoregulación [13].

El software matemático MATLAB fue utilizado en el análisis para resolver el modelo matemático. Se utiliza la herramienta de ecuaciones diferenciales parciales para emplear las técnica de elementos finitos con el fin de resolver las ecuaciones diferenciales acopladas para las cinco capas de la piel sometidas a apropiadas condiciones de contorno [14].

\section{Resultados}

Como volumen de control se consideró un sólido de $15 \times 12 \times 10.5 \mathrm{~mm}$ el cual replica un fragmento de la piel. Incrustado en el sólido coexiste un melanoma maligno invasivo de $2 \mathrm{~mm}$ de diámetro y $0.5 \mathrm{~mm}$ de profundidad.
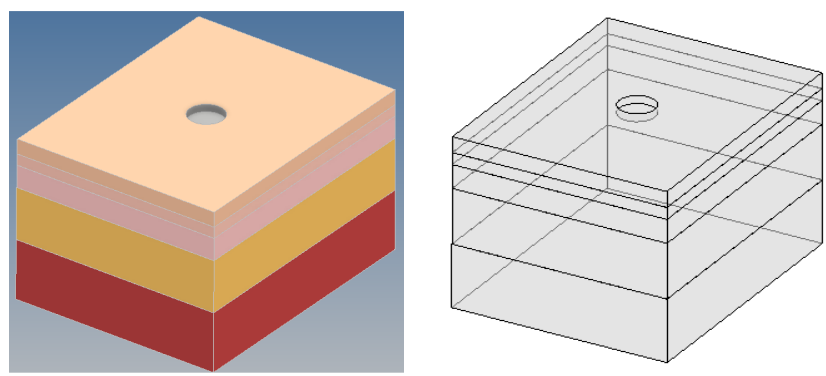

Figura 5. Diseño del volumen de control en Inventor Professional y exportado a MATLAB.

La solución propuesta por MATLAB para la ecuación de calor se da por métodos numéricos al discretizar el sólido por elementos finitos, para esto es necesario el planteamiento de una malla basada en triangulaciones.

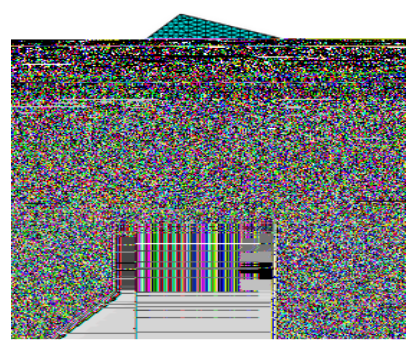

Figura 6. Mallado del sólido basado en triagulaciones.

Las cuatro paredes del sólido se asumen como adiabáticas, la parte superior del volumen se encuentra con un flujo de calor convectivo dado por $q_{\text {conv }}=h\left(T_{\infty}-T\right)$ donde $q_{c o n v}$ es el flujo de calor disipado por la superficie, $T_{\infty}$ es la temperatura del medio igual a $297 K, T$ es la temperatura promedio de la superficie de la piel igual a $303 K$ y $h$ es el coeficiente convectivo, dado por el caso de estudio, es igual a $10 \mathrm{~W} / \mathrm{m}^{2} \mathrm{~K}$ La parte inferior del volumen se mantiene en un estado estacionario a una temperatura de $310 \mathrm{~K}$ (temperatura de la sangre).

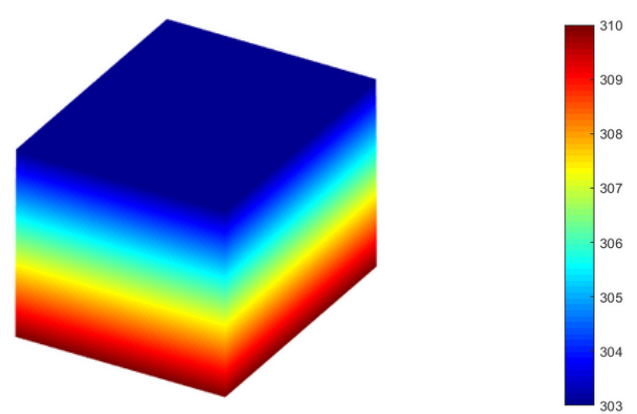

Figura 7. Análisis térmico del volumen de control para condiciones iniciales. 
Dado que el tumor no se puede detectar sin el efecto de enfriamiento, el área de la piel que contiene la lesión se enfría durante un minuto. En esta segunda etapa se cambia la condición de frontera en la superficie del volumen de control a una temperatura constante de $286 \mathrm{~K}$ con el motivo de simular el enfriamiento aplicado a la lesión.
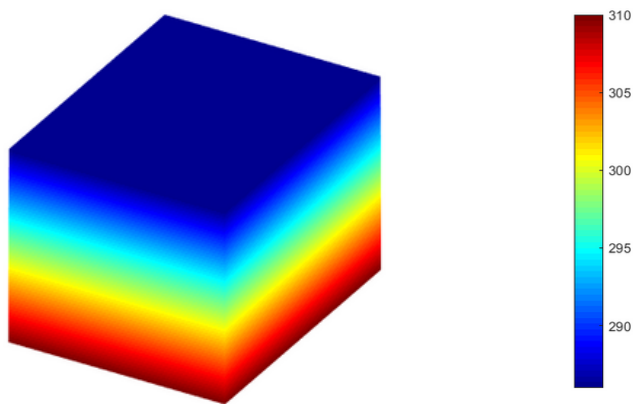

Figura 8. Análisis térmico del volumen de control durante el efecto de enfriamiento.

En la simulación de la etapa de recuperación, se plantean las condiciones de la primera etapa con la diferencia de que las condiciones iniciales de distribución de temperatura son las obtenidas por la segunda etapa.
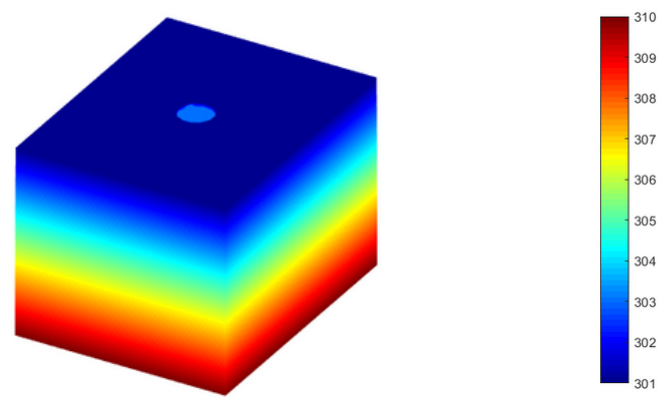

Figura 9. Análisis térmico del volumen de control después de la termoregulación.

La simulación muestra una significativa diferencia de temperatura entre el tejido sano y el centro de la lesión durante la recuperación térmica (figura 5). Este resultado apoya la hipótesis de que el aumento de la actividad metabólica de la lesión del melanoma puede ser detectado por termistores, puesto que la diferencia en la respuesta térmica entre el tejido sano y el maligno es significante.

La respuesta de la lesión maligna en la fase de termorregulación muestra una temperatura de $304 \mathrm{~K}$; en cambio, el tejido sano presenta una temperatura promedio de $301 \mathrm{~K}$. Esto indica que el melanoma se termo regula en un intervalo de tiempo menor que la piel sana.

\section{Conclusiones}

Los resultados muestran que la respuesta térmica proporciona información valiosa, el tiempo de la termorregulación es crítico para la identificación de los parámetros claves de la lesión.

El diseño, que se basa en el contacto físico con termistores, en comparación con el método de detección por imágenes térmicas dinámicas, es una alternativa de bajo costo para la posible detección de melanomas en la piel.

Gracias al tipo de termistores económicos empleados, el prototipo está diseñado para obtener con precisión diferencias de hasta 0.6 grados debido a su factor de precisión de $\pm 0.5 \%$.

En consecuencia, demostramos que es útil para tomar medidas precisas de la temperatura corporal en la dermis con el fin de lograr la detección del cáncer de piel utilizando la metodología de enfriamiento del área de interés de la piel.

Por lo tanto, así como se puede medir la actividad metabólica de un melanoma por imágenes térmicas dinámicas, la lesión puede llegar a ser detectada por nuestro prototipo basado en sensores de temperatura por resistencia.

\section{Planes Futuros}

El objetivo es optimizar el prototipo, con el fin de iniciar pruebas médicas a pacientes para validar el funcionamiento del dispositivo según la simulación realizada, a fin de que el dispositivo llegue a ser validado como una herramienta de bajo costo para contribuir al diagnóstico del cáncer de piel.

Además, se investigará en qué otras regiones del cuerpo el dispositivo puede ser utilizado para detectar de manera cuantitativa el cáncer; por ejemplo, las tiroides, la cual es una glándula ubicada en el cuello.

\section{REFERENCIAS}

[01] Mayo, M. "Cáncer de piel, ¿cómo prevenirlo?” Ministerio de Salud de la República de Panamá. [Online]. Disponible en: http://minsa.gob.pa/noticia/cancer-de-piel-como-prevenirlo [2018].

[02] Castro A. "Cierre de la XV Campaña de Concienciación, Prevención, Detección Temprana del Cáncer de Piel y Melanoma". Instituto Oncológico Nacional. [Online]. Disponible en: http://www.ion.gob.pa/cierre-de-la-xv-campanade-concienciacion-prevencion-deteccion-temprana-del-cancerde-piel-y-melanoma-2016/ [2016].

[03] Castro A. "Culmina XVI Campaña de Prevención y Detección Temprana del Cáncer de Piel”. FUNDACÁNCER. [Online]. Disponible en: https://www.fundacancerpanama.org/culminaxvi-campana-de-prevencion-y-deteccion-temprana-de-cancerde-piel/ [2017].

[04] Castro A. "Cierre XVII Campaña de Prevención y Detección Temprana del Cáncer de Piel”. FUNDACÁNCER. [Online]. Disponible en: https://www.fundacancerpanama.org/cierrexvii-campana-de-prevencion-y-deteccion-temprana-de-cancerde-piel/ [2018].

[05] Gellet A., Swetter S., Brooks K., Demierre M. y Yaroch A. "Screning early detection and trends for melanoma: current status and future directions". JAAD, 57(4), 555-572. (2007)

[06] Herman C. y Centigul M. P. Visualización y detección cuantitativa de cáncer de piel utilizando imágenes térmicas dinámicas. J. Vis. Exp., 51(2679), 1-4. (2011) 
[07] Adafruit. DHT22 temperature-humidity sensor + extras. Adafruit. [Online]. Disponible en: https://www.adafruit.com/product/385 [2018][08] Arduino. "Arduino IDE 1.8.7". [Software]. Disponible en: https://www.arduino.cc/en/Main/Software.

[09] Mathworks Inc. (2018). "MATLAB r2018b" [Software]. Disponible. en: https://la.mathworks.com/academia/student_version.html?s_tid =hp_ff_p_studen.t

[10] Autodesk Inc. (2018). "Inventor Professional 2018" [Software]. Disponible en: https://www.autodesk.com/education/freesoftware/inventor-professional.
[11] F.I. Greene, C.C. Compton, A.G. Fritz, J.P Shah, D.P. Winchester. "Melanoma of the Skin". AJCC Cancer Staging Atlas Part V. Springer, pp. 207-216. (2006).

[12] H. H. Pennes. "Analysis of tissue and arterial blood temperature in the resting human forearm". J. Appl. Physiol. 1. 93-122. (1948).

[13] M. Pirtini Centigul, C. Herman, R.M. Alani. "A heat transfer model of skin tissue for the detection of lessions: sensitivity analysis”. Phys. Med. Biol. 55. 5933-5951. (2009).

[14] MathWorks Inc. (2018). Partial Differential Equation Toolbox for Thermal Analysis (3.0) [Toolbox]. Disponible en: https://la.mathworks.com/products/pde.html?requestedDomain. 